\title{
Orientación al rol de género y uso de tabaco y alcohol en jóvenes de Morelos, México
}

\author{
Rubén Chávez-Ayala, M en Psic Soc,(') Leonor Rivera-Rivera, M en C,(') Ahideé Leyva-López, M en C,(1) \\ Marcela Sánchez-Estrada, M en Psic, ${ }^{(2)}$ Eduardo Lazcano-Ponce, PhD.(1)
}

\author{
Chávez-Ayala R, Rivera-Rivera L, Leyva-López A, \\ Sánchez-Estrada M, Lazcano-Ponce E. \\ Orientación al rol de género y uso de tabaco \\ y alcohol en jóvenes de Morelos, México. \\ Salud Publica Mex 2013;55:43-56.
}

\begin{abstract}
Resumen
Objetivo. Cuantificar la asociación entre orientación al rol de género y uso de tabaco y alcohol en jóvenes del estado de Morelos. Material y métodos. Estudio transversal realizado en 2004-2005, en jóvenes de 14 a 24 años $(n=1$ 730). Variables sociodemográficas (zona de residencia, nivel socioeconómico); familiares (educación y violencia paternal); psicosociológicas (rol de género, autoestima, depresión, consumo de alcohol, tabaco, locus de control, abuso sexual). Análisis por regresión logística. Resultados. Factores asociados con uso de tabaco: en mujeres, andrógina, no deseable, machista, intento de abuso sexual y zona urbana. Para hombres, depresión y sumisión. Factores asociados con uso de alcohol: en mujeres, rol masculino, y hombres, edad más de 20 años, vivir en zona semiurbana y urbana y locus interno. Conclusiones. El machismo es una de las orientaciones al rol de género con mayor asociación con el uso de tabaco principalmente en las jóvenes, y la orientación masculina o instrumental se asocia con el alcohol.
\end{abstract}

Palabras clave: identidad de género; alcoholismo; tabaquismo; dependencia; jóvenes; control de medicamentos y narcóticos; vulnerabilidad en salud; género y salud
Chávez-Ayala R, Rivera-Rivera L, Leyva-López A, Sánchez-Estrada M, Lazcano-Ponce E.

Gender role orientation and tobacco and alcohol use among youth in Morelos, Mexico. Salud Publica Mex 2013;55:43-56.

\begin{abstract}
Objective. To quantify the association between gender role orientation and tobacco and alcohol use among young people of the State of Morelos. Materials and methods. Study conducted in 2004-2005, students aged I4 to 24 years $(n=$ I 730). Sociodemographic variables (area of residence, socioeconomic status), family (parental education and violence), psycho-sociological (gender role, self-esteem, depression, alcohol consumption, tobacco, locus of control, sexual abuse). Logistic regression analysis. Results. Factors associated with use of tobacco: In women, being androgynous undesirable, masculine role, attempted sexual abuse and urban areas. For men, depression and submission. Factors associated with alcohol use: In women, masculine gender role; and in men to be older than 20 years, living in semi-urban and urban area, and internal locus. Conclusions. The machismo is one of the gender role orientations with greater association with the use of tobacco primarily in girls in Mexico, and the masculine or instrumental role with alcohol.
\end{abstract}

Key words: gender identity; alcoholism; smoking; dependence; young; drug and narcotic control; health vulnerability; gender and health

(I) Centro de Investigación en Salud Poblacional, Instituto Nacional de Salud Pública. Cuernavaca, Morelos, México

(2) Secretaría Académica, Instituto Nacional de Salud Pública. Cuernavaca, Morelos, México

Fecha de recibido: 7 de octubre de 201I - Fecha de aceptado: 16 de octubre de 2012

Autor de correspondencia: Mtra. Leonor Rivera Rivera. Departamento de Investigación en Salud Perinatal,

Dirección de Salud Reproductiva, Centro de Investigación en Salud Poblacional.

Av. Universidad 655, col. Santa María Ahuacatitlan. 62100 Cuernavaca, Morelos, México

Correo electrónico: Irivera@insp.mx 
Pe ara los programas de intervención en salud pública es importante incorporar conceptos dinámicos del género para comprender comportamientos de inicio y mantenimiento de las adicciones en población joven, que constituyen un problema con impacto negativo en distintas áreas de la salud, tanto en el desarrollo de enfermedades crónicas, mortalidad prematura, ${ }^{1}$ violencia y accidentes, ${ }^{2}$ como en la salud reproductiva. La gravedad es mayor cuando el consumo inicia a temprana edad y la persona incorpora a su personalidad papeles de género asociados con el consumo de sustancias, que aumentan la probabilidad de desarrollar una adicción regular y motivan a una creciente aceptación y experimentación con sustancias entre los jóvenes. ${ }^{3,4}$ Con el consumo temprano se integran además comportamientos adictivos a la personalidad cuando se controlan estados emocionales como ansiedad, miedo y depresión, ${ }^{5}$ lo que hace más difícil el abandono del consumo en la etapa adulta, por lo que para elaborar programas de intervención de mayor impacto es importante generar información que incorpore variables sociales, cognitivas y emocionales en la investigación.

En México se han realizado investigaciones desde los años setenta que señalan tendencias a la disminución en la edad de inicio en el consumo de tabaco y alcohol, e incremento de su uso en población femenina. ${ }^{6-9} \mathrm{Si}$ bien en general prevalece más el consumo de sustancias en hombres, se han encontrado algunas prevalencias mayores en mujeres. ${ }^{10,11}$ Esta tendencia también se señala en otros países. ${ }^{12-16}$

De acuerdo con la Encuesta Nacional de Adicciones de 2008, en el ámbito nacional se reportó un promedio de 13.7 años en el inicio de consumo de tabaco y en Morelos de 17.5 años, teniendo como razones más importantes de inicio curiosidad y convivencia con fumadores. En cuanto al alcohol, el consumo diario para los hombres es el mismo que el promedio nacional, $1.5 \%$ y para las mujeres es significativamente más alto, $0.5 \%$. El nivel de consumo alto en ambos está por encima del promedio nacional y el abuso/dependencia es mayor que el promedio nacional. ${ }^{17}$

Lo anterior resulta preocupante porque existen diferencias metabólicas en el procesamiento y eliminación de sustancias que generan mayores riesgos de daños a la salud en general y a la salud reproductiva en la mujer. En el caso del alcohol, su consumo excesivo se asocia con aborto espontáneo, ${ }^{18}$ retraso mental y defectos de nacimiento; ${ }^{19,20}$ preeclampsia y desprendimiento de placenta, ${ }^{21}$ cáncer de mama ${ }^{22}$ y otros tipos de cánceres. ${ }^{23}$ En el caso del tabaco, la mujer con consumo excesivo tiene más riesgo de contraer padecimientos

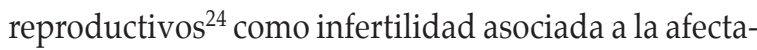
ción de actividad hormonal, ${ }^{25}$ retrasos conceptivos, ${ }^{26}$ daños al ovario y menopausia temprana, ${ }^{27}$ riesgo de embarazo ectópico, ${ }^{28}$ aborto y parto pretérmino, ${ }^{29,30}$ riesgo de placenta previa, ${ }^{27}$ desprendimiento de la misma, ${ }^{21}$ patología vascular de placenta, ${ }^{31}$ bajo peso del producto, ${ }^{32}$ incremento de riesgo de muerte súbita en el lactante ${ }^{33}$ y otros riesgos más conocidos como cáncer pulmonar, de mama, arterioesclerosis, hipertensión arterial, infarto al miocardio y enfermedades respiratorias. ${ }^{34}$ En hombres, el consumo excesivo de alcohol afecta aspectos de salud reproductiva como la morfología de espermatozoides y producción de esperma. ${ }^{35,36}$ Además, el exceso en consumo de tabaco se asocia con disfunción eréctil. 37,38

Por otra parte, en cuanto al desarrollo de las adicciones, diversas variables culturales y psicosociales influyen en la adopción de estos comportamientos. ${ }^{39}$ Dentro de estas se encuentran los estereotipos de roles de género, orientación al rol de género y comportamientos asociados con el género, que se expresan en la autoimagen, estilos de afrontamiento, comportamiento violento o de sumisión, funcionamiento social y desarrollo de depresión y ansiedad, entre otros. ${ }^{40-43}$ Se ha señalado que el consumo de tabaco y alcohol está ligado a los papeles de género y predomina su consumo en hombres. Se desconoce si en México, al ganar la mujer mayores derechos, salario y flexibilización de las normas de género, ha incorporado estas adicciones a su estilo de vida, como ha sido señalado en otros países. ${ }^{44}$

En la década de los ochenta, Bem postula dentro de la corriente cognoscitiva de la psicología la "teoría del esquema de género" ${ }^{\prime 45}$ que explica que los roles de género provienen del contexto sociocultural y son un principio de organización social, incorporado a la personalidad mediante un "esquema cognoscitivo" que organiza la percepción. Este esquema incluye información sobre diferencias anatómicas, funciones reproductivas, división sexual del trabajo y atributos de la personalidad. El esquema orienta sobre qué atributos y comportamientos deben estar relacionados con el sexo biológico y la identidad personal y se construye a partir de la influencia de la cultura de género familiar, que incluye la violencia intrafamiliar como expresión de relaciones de poder, la educación, el modelaje social y reforzamientos o castigos en las relaciones interpersonales.

Poco se sabe de la contribución de la orientación al rol de género en el consumo de sustancias en México, pues los programas de intervención están basados en estereotipos de género dirigidos hacia población mas- 
culina. Estos resultados pueden contribuir a redirigir las intervenciones desde un enfoque de género. El objetivo del presente estudio es cuantificar la fuerza de asociación entre la orientación al rol de género y el uso de tabaco y alcohol en el estado de Morelos, México, con el fin de que se pueda incorporar este conocimiento a programas de salud pública.

\section{Material y métodos}

\section{Diseño y población de estudio}

El presente estudio es transversal, correlacional y multivariado y forma parte de una cohorte. ${ }^{46}$ En este estudio participaron 1730 estudiantes inscritos durante el periodo escolar 2004-2005 en escuelas de nivel medio superior, universidades e institutos tecnológicos. Organizados en pequeños grupos en las escuelas, se utilizó un cuestionario autoaplicable para recabar la información; el tiempo de aplicación promedio fue de 40 minutos. El cuestionario tuvo las siguientes secciones: a) factores sociodemográficos, (sexo, edad, zona de residencia, nivel socioeconómico); b) factores familiares, (nivel educativo de los padres, violencia entre padres); c) factores psicológicos individuales, (orientación al rol de género, autoestima, depresión, locus de control interno y externo).

El protocolo de este estudio fue aprobado por el Comité de ética, investigación y bioseguridad del Instituto Nacional de Salud Pública. A los estudiantes se les entregaron cartas de consentimiento informado y de autorización para los padres, cuando el estudiante era menor de edad. Si eran mayores de edad, antes de contestar la encuesta se les pidió que aceptaran participar en el estudio firmando la carta de asentimiento. En ambos casos, se explicaron en detalle los objetivos del estudio, confidencialidad y contenido del cuestionario.

\section{Medición de las variables}

Factores sociodemográficos

Edad

En cuanto a la edad se dividió a la muestra a partir de la media (19.8) para analizarla en dos grupos de edad: a) mayores o iguales a los 20 años y b) menores de 20 años.

\section{Nivel socioeconómico}

El nivel socioeconómico se construyó utilizando el índice de Bronfman y cols. ${ }^{47}$ desde la primera medición de esta cohorte en 1999. Aunque existen índices más actualizados, su modificación en la tercera medición no haría comparable esta variable con la obtenida en las dos etapas previas del estudio. Este índice se construyó a partir de las siguientes variables: a) material del piso de la vivienda; $b$ ) disponibilidad de agua entubada; $c$ ) infraestructura para la eliminación de excretas; d) número de personas que habitan la vivienda; e) número de cuartos, y f) escolaridad del (o la) jefe de familia.

Zona de residencia

Se clasificó en urbana, semiurbana y rural tomando en cuenta los criterios demográficos del Instituto de Estadística y Geografía de México. ${ }^{48}$

\section{Factores familiares}

Nivel de escolaridad de los padres

El máximo nivel de escolaridad de los padres se categorizó por ciclos educativos incompletos y completos: $\sin$ escolaridad, primaria incompleta y completa, secundaria incompleta y completa, carrera técnica incompleta y completa, preparatoria incompleta y completa, universidad (o equivalente técnico) incompleta y completa. Se agruparon como: sin escolaridad, primaria-secundaria, preparatoria y universidad, creando cada grupo como una variable dummy.

Violencia entre padres

Esta variable se construyó mediante cuatro preguntas: "¿Tu padre insulta u ofende a tu mamá?", "¿Tu papá golpea a tu mamá?", "¿Tu madre insulta u ofende a tu papá?", y “¿Tu mamá golpea a tu papá?".

\section{Factores individuales}

\section{Consumo diario de tabaco}

Este criterio de consumo diario de tabaco se tomó de la Encuesta Global de Tabaquismo en Adultos $2009^{49}$ y para medirlo se utilizaron dos preguntas: 1) ¿Tú fumas cigarrillos? Con la opción de respuesta "Si", y 
2)¿Cuántos cigarros te fumas al día?, con las opciones: a) sólo algunas fumadas, b) de 1 a 2; c) de 3 a 5; d) de 6 a 10, e) más de 10 . Se consideró como consumo diario de tabaco cuando la persona dijo que fumaba y señaló cualquiera de las opciones de respuesta de la pregunta 2. Además se preguntó el tiempo de haber empezado a fumar en meses y consumo en el último mes.

Uso regular de alcohol

Se hicieron cuatro preguntas: 1) ¿Consumes bebidas alcohólicas? 2) ¿Hace cuánto empezaste a tomar bebidas alcohólicas? 3) ¿Con qué frecuencia consumes bebidas alcohólicas? 4) En los últimos doce meses ¿qué tan seguido tomaste alguna bebida que contenga alcohol? 5) En los últimos doce meses icon qué frecuencia te sentiste embriagado, con dificultad para mantenerte en pie o para caminar? El "consumo regular de alcohol" se construyó a partir de la pregunta 2 incluyendo desde "consumo diario" hasta "una vez a la semana". ${ }^{50}$

Orientación al rol de género

Se midieron ocho orientaciones al rol de género utilizando el Inventario de Masculinidad y Feminidad $(\mathrm{IMAFE})^{51}$ que genera dos primeras dimensiones llamadas: "masculinidad", que mide la orientación a metas ligadas a la función de sostén económico y protección del hogar y la familia, ${ }^{52}$ y "feminidad", que mide la expresión de emociones ligada a la función de cuidado y soporte emocional familiar que, según su autora, son roles de género "deseables" desde la perspectiva cultural tradicional. Cuando ambos rasgos se presentan con puntajes altos clasificamos a las personas como "andróginas deseables". También están las escalas: "machismo", que mide comportamientos agresivos de prepotencia y dominación; y "sumisión", que mide el dejarse dominar por otra persona. Según la autora, estos rasgos son culturalmente "no deseables". Cuando se presentan juntos les clasificamos como "andróginos no deseables", que son personas que pueden ser dominantes y agresivas hacia unas personas y sumisas con otras. Por ejemplo, el hombre que es sumiso ante el jefe y dominante con la esposa.

Estas dimensiones son medidas en una escala de siete grados que expresa la frecuencia del rasgo, desde "nunca o casi nunca soy asi" hasta "siempre o casi siempre soy asi". Se recodificaron como variables dummy: 1 para los que tenían los niveles altos en cada dimensión y 0 para los que presentaron los niveles bajos.
Autoestima

La autoestima se midió por el Inventario de Autoestima de Coopersmith. ${ }^{53}$ Por análisis factorial se obtuvo un primer factor $(\alpha=0.84)$ al que se denominó "autoestima baja".

\section{Depresión}

Se midió con la escala de Zung, ${ }^{54}$ la cual fue analizada con el método de componentes principales. Se encontró un factor principal que contiene 10 reactivos $(\alpha=0.87)$.

Locus de control

Se utilizó la escala de locus de control. ${ }^{55}$ Esta escala mide creencias de causalidad sobre acontecimientos en la vida de las personas. Se evaluaron solamente las dimensiones de locus de control interno y externo.

Locus de control interno

Se midió por los siguientes reactivos: "El que yo llegue a tener éxito depende de mí", "mis calificaciones dependen de mi esfuerzo", "el que yo consiga un buen empleo depende de mis capacidades", y "que obtenga las cosas que quiero depende de mí" $(\alpha=0.89)$.

Locus de control externo

Se midió por los siguientes reactivos: "mi país está dirigido por pocas personas en el poder y lo que yo haga no cambia nada", "las guerras dependen de los gobiernos y no hay mucho que yo pueda hacer", "el problema del hambre está en manos de los poderosos y no hay mucho que yo pueda hacer al respecto", "los problemas mundiales están en manos de los poderosos y lo que yo haga no cambia nada" $\mathrm{y}$ "no puedo influir en la solución de problemas de vivienda ya que depende del gobierno" $(\alpha=0.86)$.

\section{Víctima de abuso sexual}

Se definió "abuso sexual" según Wyatt y Russell, ${ }^{56,57}$ pero se desconoció edad del agresor. Se consideró "intento de violación" como un conato de abuso sexual; y "abuso sexual consumado", a la violación sexual consumada en menores de 18 años. Se usaron dos preguntas: “¿Alguna vez intentaron obligarte a tener relaciones 
sexuales?" y "¿Alguna vez te han obligado a tener relaciones sexuales en contra de tu voluntad?". Con dos variables de respuesta: "intento de abuso sexual" (cuando la persona respondió afirmativamente a la pregunta sobre intento y negativamente a la de "abuso consumado") y "abuso sexual consumado" (cuando la persona respondió afirmativamente a la pregunta sobre "abuso sexual consumado", independientemente de cómo haya contestado a la pregunta sobre "intento").

\section{Análisis estadístico}

Se realizaron análisis de frecuencias por variable para conocer su distribución y valores aberrantes. Posteriormente se realizaron análisis bivariados de ji cuadrada y regresión logística. Para el análisis multivariado se construyeron modelos de regresión logística múltiple saturados y se ajustó por efectos de diseño de la encuesta. Para el modelo final se identificaron las variables independientes que fueron significativas del análisis bivariado en su asociación con uso de tabaco y alcohol $(p<0.05)$. La prueba de bondad de ajuste se evaluó mediante la prueba Hosmer-Lemeshow. ${ }^{58} \mathrm{El}$ análisis se realizó por medio de los paquetes estadísticos de Stata v9.0.

\section{Resultados}

De los 1730 estudiantes estudiados 60.4\% fueron mujeres. El promedio de edad fue de 19.82 con un intervalo de 16 a 24 años; $68.8 \%$ pertenecía a los niveles socioeconómicos bajo y medio. En relación con el tipo de localidad 34.2\% fueron residentes del área rural. Se encontró una prevalencia general de consumo regular de alcohol de $5.4 \%$ y de consumo diario de cigarrillo de $23.9 \%$, de los cuales $38.4 \%$ reportó fumar "sólo algunas fumadas diario".

En el cuadro I se describen características de las variables investigadas por género. Las mujeres reportan mayor prevalencia de insultos entre los padres, más depresión y victimización por abuso sexual que los hombres. A su vez, estos presentaron menor autoestima y un locus externo mayor que las mujeres. En cuanto a las diferencias en orientación al rol de género, las mujeres tuvieron mayor prevalencia en "feminidad" que los hombres y éstos mayores prevalencias en "masculinidad" y "machismo". En la dimensión "androginia deseable", los hombres se orientan más tanto al logro de metas (masculinidad), como a la expresividad emocional (feminidad) que las mujeres. También hay una orientación mayor a la "androginia no deseable" en hombres, es decir "machismo" y "sumisión" conjuntos $(p<0.05)$.

\section{Uso regular de alcohol y tabaco}

En los cuadros II y III se describen características del uso diario de tabaco y alcohol regular por género y variables investigadas. Las mujeres en zonas urbanas fumaron más, y conforme aumenta la escolaridad del padre, el consumo de tabaco aumenta también. La depresión fue mayor en fumadoras diarias así como el locus de control externo, que expresa vulnerabilidad a la presión social. Intento y violación consumada son más frecuentes entre fumadoras, no así la violencia entre padres. En hombres, los de mayor edad fuman más, así como los altos en depresión.

El consumo diario de tabaco en hombres fue $43 \%$ mayor que en las mujeres $(p<0.001)$; el inicio temprano $42 \%$ mayor $(p<0.001)$ y el consumo en el último mes $70.3 \%$ mayor $(p<0.05)$. A su vez, las mujeres más jóvenes iniciaron el uso de tabaco $72.9 \%$ más temprano que las mayores ( $p=0.03$ ).

El consumo regular de alcohol fue mayor en hombres $(p<0.001)$ y lo consumieron con mayor frecuencia semanal, quincenal y mensualmente $(p<0.001)$. También en el consumo de alcohol hasta la embriaguez los hombres presentaron mayores prevalencias en todas las categorías $(p<0.05)$. No se encontraron diferencias significativas por género en promedio de meses de inicio en el consumo.

\section{Factores asociados con el uso de tabaco}

El cuadro IV muestra el análisis de regresión logística múltiple. En mujeres, las variables que incrementaron la probabilidad de consumo diario de tabaco fueron las sociodemográficas: vivir en zona urbana; individuales: haber tenido una experiencia de intento de abuso sexual; la orientación a los roles "machista" y "andrógina no deseable" (machismo y sumisión conjuntos), lo que señala que el cambio en los roles de género y el consumo de tabaco en las mujeres es un fenómeno urbano. En hombres, de los factores individuales, presentar depresión alta incrementó el consumo diario de tabaco y ser sumiso lo disminuyó. Tampoco se encontró asociación con violencia entre padres.

\section{Factores asociados con el consumo regular de alcohol}

En el cuadro V se presenta el análisis de regresión logística múltiple. En mujeres, se encontró que de los factores individuales, la orientación a la masculinidad se asoció con el consumo de alcohol. En hombres, de los factores sociodemográficos se asociaron tener más de 20 años, 
Cuadro I

Características de las variables de estudiantes de escuelas públicas por género. Morelos, México, 2004-2005

\begin{tabular}{lcccc}
$\begin{array}{l}\text { Total \% } \\
(n=1730)\end{array}$ & $\begin{array}{c}\text { Hombres \% } \\
(n=678)\end{array}$ & $\begin{array}{c}\text { Mujeres \% } \\
(n=1042)\end{array}$ & Valor $p$ \\
$\begin{array}{lcccc}\text { Edad } \\
16-17\end{array}$ & 21.6 & 22.2 & 21.2 & \\
\hline $18-19$ & 29.2 & 26.5 & 30.9 & \\
\hline $20-21$ & 24.9 & 24.4 & 25.1 & \\
\hline 22 o más & 24.4 & 26.9 & 22.7 & 0.114 \\
& & & & \\
Estado civil & & & & \\
Soltero & 94.0 & 95.3 & 93.2 & \\
\hline Casados & 3.1 & 1.8 & 4.0 & \\
\hline Separados & 0.9 & 1.0 & 0.9 & \\
\hline Divorciados & 0.2 & 0.3 & 0.1 & \\
\hline Viudo & 0.1 & 0 & 0.1 & 0.128
\end{tabular}

Ocupación

\begin{tabular}{lcccc} 
Estudiante & 94.6 & 95.1 & 94.3 & \\
\hline Estudiante y trabajador & 3.4 & 3.4 & 3.4 & \\
\hline Ama de casa & 0.2 & 0 & 0.3 & \\
\hline Empleado & 1.4 & 1.5 & 1.4 & \\
\hline Estudiante y hogar & 0.2 & 0 & 0.3 & \\
\hline Buscando trabajo & 0.2 & 0 & 0.4 & 0.225
\end{tabular}

Características familiares

Escolaridad del padre

\begin{tabular}{lcccc}
\hline Sin educación & 6.3 & 7.1 & 5.8 & \\
\hline Nivel primaria & 28.5 & 28.1 & 28.7 & \\
\hline Nivel secundaria & 24.6 & 26.7 & 25.8 & \\
\hline Preparatoria o más & 40.2 & 38.9 & 39.4 & 0.545
\end{tabular}

Escolaridad de la madre

\begin{tabular}{lcccc} 
Sin educación & 7.4 & 9.2 & 6.2 & \\
\hline Nivel primaria & 31.0 & 28.1 & 32.9 & \\
\hline Nivel secundaria & 29.2 & 29.7 & 28.8 & \\
\hline Preparatoria o más & 32.4 & 33.0 & 32.1 & 0.045
\end{tabular}

Violencia intrafamiliar

Padre golpea a la madre

\begin{tabular}{|c|c|c|c|c|}
\hline Sí & 3.9 & 3.3 & 4.2 & \\
\hline No & 96.7 & 96.7 & 95.8 & 0.220 \\
\hline \multicolumn{5}{|c|}{ Madre golpea al padre } \\
\hline Sí & 1.2 & 1.0 & 1.3 & \\
\hline No & 98.8 & 99.0 & 98.7 & 0.334 \\
\hline \multicolumn{5}{|c|}{ Padre insulta a la madre } \\
\hline Sí & 17.8 & 14.5 & 19.9 & \\
\hline No & 82.2 & 85.5 & 80.1 & 0.003 \\
\hline \multicolumn{5}{|c|}{ Madre insulta al padre } \\
\hline Sí & 11.7 & 9.7 & 13.0 & \\
\hline No & 88.3 & 90.3 & 87.0 & 0.027 \\
\hline
\end{tabular}

Factores psicosociales

Uso diario de tabaco

\begin{tabular}{lrrrr} 
Sí & 24.9 & 33.7 & 19.2 & \\
\hline $\begin{array}{l}\text { No } \\
\text { Uso regular de alcohol }\end{array}$ & 75.1 & 66.3 & 80.8 & 0.000 \\
Sí & 13.4 & 17.3 & 9.7 & \\
\hline No & 13.4 & 82.7 & 90.3 & 0.002
\end{tabular}

\begin{tabular}{|c|c|c|c|c|}
\hline \multicolumn{5}{|c|}{ Frecuencia consumo de alcohol } \\
\hline Diario & 4.0 & 0.3 & 6.0 & \\
\hline Cada ocho días & 12.9 & 17.0 & 9.2 & \\
\hline Cada quince días & 15.8 & 20.7 & 11.4 & \\
\hline Cada mes & 26.6 & 28.0 & 25.3 & \\
\hline 2 a 5 veces al año & 36.8 & 30.7 & 42.3 & \\
\hline Una vez al año & 7.4 & 3.3 & 11.1 & 0.000 \\
\hline
\end{tabular}

Orientación al rol de género

Masculinidad

\begin{tabular}{lcccc} 
Bajo & 90.5 & 88.5 & 91.8 & \\
\hline Alto & 9.5 & 11.5 & 8.2 & 0.013 \\
\hline $\begin{array}{l}\text { Feminidad } \\
\text { Bajo }\end{array}$ & & & & \\
\hline Alto & 76.2 & 83.7 & 71.3 & \\
\hline & 23.8 & 16.3 & 28.7 & 0.000
\end{tabular}

Androginia

Deseable

\begin{tabular}{lllll} 
Bajo & 70.7 & 66.1 & 73.7 & \\
\hline Alto & 29.3 & 33.9 & 26.3 & 0.000 \\
$\begin{array}{l}\text { Indiferenciado } \\
\begin{array}{l}\text { Deseable } \\
\text { Bajo }\end{array}\end{array}$ & 68.1 & 67.9 & 68.5 & \\
\hline Alto & 31.9 & 32.1 & 31.5 & 0.411 \\
\hline $\begin{array}{l}\text { Machismo } \\
\text { Bajo }\end{array}$ & 74.9 & 71.7 & 76.9 & \\
\hline Alto & 25.1 & 28.3 & 23.1 & 0.010 \\
\hline $\begin{array}{l}\text { Sumisión } \\
\text { Bajo }\end{array}$ & 55.6 & 54.2 & 56.5 & \\
\hline Alto & 44.4 & 45.8 & 43.5 & 0.179 \\
\hline $\begin{array}{l}\text { Androginia } \\
\text { No deseable } \\
\text { Bajo }\end{array}$ & 71.9 & 69.2 & 73.7 & \\
\hline Alto & 28.1 & 30.8 & 26.3 & 0.025 \\
\hline $\begin{array}{l}\text { Indiferenciado } \\
\text { No deseable }\end{array}$ & & & & \\
Bajo & 38.2 & 38.9 & 37.8 & \\
\hline Alto & 61.8 & 61.1 & 62.2 & 0.343 \\
\hline $\begin{array}{l}\text { Autoestima } \\
\text { Baja }\end{array}$ & 62.4 & 67.1 & 59.5 & \\
\hline Alta & 37.6 & 32.9 & 40.5 & 0.001 \\
\hline $\begin{array}{l}\text { Depresión } \\
\text { Baja }\end{array}$ & 85.7 & 89.1 & 83.6 & \\
\hline Alta & 14.3 & 10.9 & 16.4 & 0.001 \\
\hline $\begin{array}{l}\text { Locus de control interno } \\
\text { Bajo }\end{array}$ & 42.4 & 46.0 & 40.1 & \\
\hline Alto & 57.6 & 54.0 & 59.9 & 0.009 \\
\hline $\begin{array}{l}\text { Externo } \\
\text { Bajo }\end{array}$ & 47.2 & 42.2 & 50.4 & \\
\hline $\begin{array}{l}\text { Alto } \\
\text { Alto }\end{array}$ & 57.8 & 49.6 & 0.001 \\
\hline
\end{tabular}

Víctima de intento de abuso sexual

\begin{tabular}{lcccc} 
Sí & 7.2 & 4.1 & 9.2 & \\
\hline No & 92.8 & 95.9 & 90.8 & 0.000
\end{tabular}

Víctima de abuso sexual consumado

\begin{tabular}{lcccc} 
Sí & 2.9 & 1.9 & 3.6 & \\
\hline No & 97.1 & 98.1 & 96.4 & 0.032
\end{tabular}


Cuadro II

Prevalencia de uso diario de tabaco en estudiantes de escuelas Públicas por diferentes factores. Morelos, México, 2004-2005

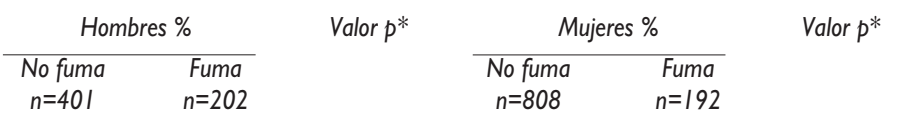

Factores sociodemográficos

\begin{tabular}{|c|c|c|c|c|c|c|c|}
\hline Zona de residencia & Rural & 40.4 & 30.7 & 0.054 & 34.2 & 23.4 & 0.005 \\
\hline & Semiurbana & 22.7 & 25.7 & & 27.5 & 26.6 & \\
\hline & Urbana & 36.9 & 43.6 & & 38.4 & 50.0 & \\
\hline \multirow[t]{2}{*}{ Edad } & Menor de 20 & 53.4 & 39.4 & 0.001 & 53.3 & 47.4 & 0.080 \\
\hline & gual a 20 años & 46.6 & 60.6 & & 46.7 & 52.6 & \\
\hline \multirow[t]{3}{*}{ Índice socioeconómico } & Bajo & 5.8 & 6.0 & $0.87 \mid$ & 2.5 & 2.6 & 0.218 \\
\hline & Regular & 90.4 & 89.4 & & 90.3 & 86.5 & \\
\hline & Alto & 3.7 & 4.6 & & 7.2 & 10.9 & \\
\hline
\end{tabular}

Características familiares

\begin{tabular}{rrrrrrrr} 
Escolaridad del padre & Muy Baja & 7.7 & 6.0 & 0.266 & 5.1 & 8.9 & 0.037 \\
\hline Baja & 30.1 & 24.3 & 30.0 & 21.4 & \\
\hline Regular & 24.0 & 24.8 & 26.4 & 27.6 & \\
\hline Alta & 38.2 & 45.0 & 38.6 & 42.2
\end{tabular}

\begin{tabular}{rrrrrrrr} 
Escolaridad de la madre & Muy Baja & 8.4 & 11.0 & 0.667 & 6.3 & 4.7 & 0.147 \\
\hline Baja & 27.3 & 28.0 & 34.4 & 27.6 & \\
\hline Regular & 29.8 & 29.8 & 28.3 & 29.7 & 38.0
\end{tabular}

Violencia familiar

\begin{tabular}{|c|c|c|c|c|c|c|c|}
\hline Padre golpea madre & Sí & 3.0 & 3.5 & 0.446 & 3.9 & 4.9 & 0.315 \\
\hline & No & 97.0 & 96.5 & & 96.1 & 95.1 & \\
\hline \multirow[t]{2}{*}{ Padre insulta a madre } & Sí & 12.6 & 16.6 & 0.115 & 20.0 & 18.7 & 0.391 \\
\hline & No & 87.4 & 83.4 & & 80.0 & 81.3 & \\
\hline \multirow[t]{2}{*}{ Madre golpea al padre } & Sí & 1.0 & 0.5 & 0.457 & 1.2 & 1.1 & 0.634 \\
\hline & No & 99.0 & 99.5 & & 98.8 & 98.9 & \\
\hline \multirow[t]{2}{*}{ Madre insulta al padre } & Sí & 8.3 & 11.9 & 0.100 & 13.4 & 11.0 & 0.231 \\
\hline & No & 91.8 & 88.1 & & 86.6 & 89.0 & \\
\hline
\end{tabular}

Factores psicosociales

\begin{tabular}{ccccccccc} 
Autoestima & Baja & 65.9 & 68.4 & 0.294 & 58.7 & 58.8 & 0.523 \\
\hline Depresión & Alta & 34.1 & 31.6 & & 41.3 & 41.2 & \\
\hline & Baja & 91.6 & 83.0 & 0.001 & 84.5 & 78.6 & 0.034 \\
\hline & Alta & 8.4 & 17.0 & & 15.5 & 21.4 &
\end{tabular}


Continuación.

\begin{tabular}{|c|c|c|c|c|}
\hline \multicolumn{2}{|c|}{ Hombres \% } & \multirow[t]{2}{*}{ Valor $p^{*}$} & \multicolumn{2}{|c|}{ Mujeres \% } \\
\hline $\begin{array}{l}\text { No Fuma } \\
n=40 I\end{array}$ & $\begin{array}{c}\text { Fuma } \\
n=202\end{array}$ & & $\begin{array}{c}\text { No Fuma } \\
n=808\end{array}$ & $\begin{array}{l}\text { Fuma } \\
n=192\end{array}$ \\
\hline
\end{tabular}

Valor $p^{*}$

Locus de control

\begin{tabular}{ccccccccc} 
Interno & Bajo & 48.3 & 43.1 & 0.124 & 40.5 & 37.0 & 0.210 \\
\hline & Alto & 51.7 & 56.9 & 59.5 & 63.0 & & & \\
Externo & & & & & & & \\
& Bajo & 43.0 & 38.9 & 0.182 & 52.0 & 43.6 & 0.023 \\
\hline & Alto & 57.0 & 61.1 & & 48.0 & 56.4 &
\end{tabular}

Orientación al rol de género

\begin{tabular}{|c|c|c|c|c|c|c|c|}
\hline Masculinidad & Baja & 87.4 & 89.0 & 0.328 & 92.2 & 90.1 & 0.207 \\
\hline & Alta & 12.6 & 11.0 & & 7.8 & 9.9 & \\
\hline \multirow[t]{2}{*}{ Feminidad } & Baja & 84.6 & 81.2 & 0.160 & 71.0 & 72.4 & 0.391 \\
\hline & Alta & 15.4 & 18.8 & & 29.0 & 27.6 & \\
\hline \multirow[t]{2}{*}{ Androginia deseable } & Baja & 68.8 & 64.2 & 0.141 & 74.8 & 70.3 & 0.122 \\
\hline & Alta & 31.2 & 35.8 & & 25.2 & 29.7 & \\
\hline \multirow[t]{2}{*}{ Indiferenciado deseable } & Baja & 65.5 & 73.9 & 0.019 & 66.7 & 72.4 & 0.144 \\
\hline & Alta & 34.5 & 26.1 & & 33.3 & 27.6 & \\
\hline \multirow[t]{2}{*}{ Machismo } & Baja & 72.0 & 70.2 & 0.344 & 78.8 & 69.8 & 0.006 \\
\hline & Alta & 28.0 & 29.8 & & 21.2 & 30.2 & \\
\hline \multirow[t]{2}{*}{ Sumisión } & Baja & 86.7 & 93.6 & 0.005 & 84.4 & 90.1 & 0.044 \\
\hline & Alta & 13.3 & 6.4 & & 15.6 & 9.9 & \\
\hline \multirow[t]{2}{*}{ Androginia no deseable } & Baja & 72.0 & 64.2 & 0.026 & 75.5 & 66.7 & 0.012 \\
\hline & Alta & 28.0 & 35.8 & 24.5 & 33.3 & & \\
\hline \multirow[t]{2}{*}{ Indiferencia no deseable } & Baja & 41.3 & 36.2 & 0.125 & 36.8 & 40.1 & 0.389 \\
\hline & Alta & 58.7 & 63.8 & & 63.2 & 59.9 & \\
\hline \multirow[t]{2}{*}{ Víctima de intento de abuso sexual } & No & 96.5 & 94.5 & 0.159 & 92.6 & 84.9 & 0.00 \\
\hline & Sí & 3.5 & 5.5 & & 7.4 & 15.1 & \\
\hline \multirow[t]{2}{*}{ Víctima de abuso sexual consumado } & No & 98.6 & 96.8 & 0.109 & 97.4 & 92.1 & 0.001 \\
\hline & Sí & 1.4 & 3.2 & & 2.6 & 7.9 & \\
\hline
\end{tabular}

habitar en zonas urbana y semiurbana, y de los factores individuales, el locus de control interno. No se encontró asociación con violencia entre padres.

\section{Discusión}

Las prevalencias de consumo diario de tabaco por género encontradas en este estudio son mayores a las de la Encuesta Global de Tabaquismo en Adultos 2009 $(\mathrm{GATS})^{49}$ en México, único estudio comparable por la clasificación de "consumo diario", que señala prevalencias nacionales de consumo de $7.6 \%$ en general; $3.7 \%$, en mujeres y $11.8 \%$, en hombres. Estas diferencias pudieran explicarse por el alto porcentaje de fumadores de "sólo algunas fumadas al día" que se encontró en este estudio. Muchos fumadores pudieran no considerarse como tales porque solo dan "algunas fumadas". Se les consideró como fumadores diarios porque la adicción está establecida aunque las cantidades de nicotina sean menores. Se trata de una población joven que en unos 


\section{Cuadro III}

Prevalencia de uso regular de alcohol en estudiantes de escuelas públicas por diferentes factores. Morelos, México, 2004-2005

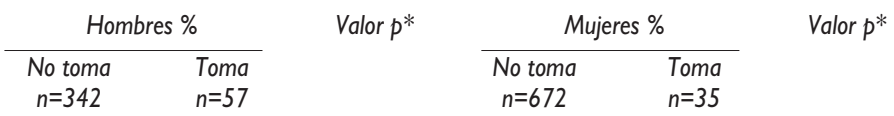

Factores sociodemográficos

\begin{tabular}{|c|c|c|c|c|c|c|c|}
\hline Zona de residencia & Rural & 46.2 & 17.5 & 0.000 & 34.6 & 22.9 & 0.053 \\
\hline & Semiurbana & 20.2 & 24.6 & 28.7 & 20.0 & & \\
\hline & Urbana & 33.6 & 57.9 & & 36.8 & 57.1 & \\
\hline \multirow[t]{2}{*}{ Edad } & Menor de 20 & 60.2 & 29.8 & 0.000 & 56.9 & 40.0 & 0.037 \\
\hline & gual a 20 años & 39.8 & 70.2 & 43.1 & 60.0 & & \\
\hline \multirow[t]{3}{*}{ Índice socioeconómico } & Bajo & 6.4 & 3.5 & 0.390 & 2.5 & 0.0 & 0.578 \\
\hline & Regular & 89.8 & 89.5 & & 91.0 & 91.4 & \\
\hline & Alto & 3.8 & 7.0 & & 6.5 & 8.6 & \\
\hline
\end{tabular}

Características familiares

\begin{tabular}{rrrrrrrr}
\hline Escolaridad del padre & Muy Baja & 6.3 & 5.3 & 0.446 & 8.2 & 5.3 & 0.870 \\
\hline Baja & 24.3 & 33.3 & 30.1 & 33.3 & \\
\hline Regular & 29.0 & 21.1 & 21.6 & 21.1 & \\
\hline Alta & 40.4 & 40.4 & 40.1 & 40.4 &
\end{tabular}

\begin{tabular}{rrrrrrrr} 
Escolaridad de la madre & Muy Baja & 9.6 & 5.3 & 0.633 & 9.4 & 5.3 & 0.790 \\
\hline Baja & 28.7 & 28.1 & 27.8 & 28.1 & \\
\hline Regular & 31.6 & 29.8 & 28.1 & 29.8 & 36.8
\end{tabular}

Violencia familiar

\begin{tabular}{|c|c|c|c|c|c|c|c|}
\hline Padre golpea madre & Sí & 3.4 & 3.6 & 0.599 & 4.1 & 6.1 & 0.408 \\
\hline & No & 96.6 & 96.4 & & 95.9 & 93.9 & \\
\hline \multirow[t]{2}{*}{ Padre insulta a madre } & Sí & 12.1 & 21.4 & 0.052 & 19.3 & 27.3 & 0.183 \\
\hline & No & 87.9 & 78.6 & & 80.7 & 72.7 & \\
\hline \multirow[t]{2}{*}{ Madre golpea al padre } & Sí & 0.6 & 1.8 & 0.379 & 1.3 & 2.9 & 0.375 \\
\hline & No & 99.4 & 98.2 & & 98.7 & 97.1 & \\
\hline \multirow[t]{2}{*}{ Madre insulta al padre } & Sí & 7.8 & 14.3 & 0.095 & 12.4 & 11.8 & 0.585 \\
\hline & No & 92.2 & 85.7 & & 87.6 & 88.2 & \\
\hline
\end{tabular}

Factores individuales

\begin{tabular}{ccccccccc} 
Autoestima & Baja & 63.3 & 71.4 & 0.151 & 59.0 & 76.5 & 0.030 \\
\hline & Alta & 36.7 & 28.6 & & 41.0 & 23.5 & \\
Depresión & Baja & 88.3 & 77.2 & 0.214 & 80.9 & 77.1 & 0.151 \\
\hline & Alta & 11.7 & 22.8 & & 19.1 & 22.9 &
\end{tabular}


Continuación...

Hombres \%

\begin{tabular}{|c|c|c|c|c|}
\hline & & \multirow[t]{2}{*}{$\begin{array}{c}\text { Mujeres \% } \\
\text { Valor } p^{*}\end{array}$} & \multicolumn{2}{|c|}{$\begin{array}{c}\text { Valor } p^{*} \\
\text { Mujeres \% }\end{array}$} \\
\hline $\begin{array}{c}\text { No toma } \\
n=342\end{array}$ & $\begin{array}{l}\text { Toma } \\
n=57\end{array}$ & & $\begin{array}{c}\text { No toma } \\
n=672\end{array}$ & $\begin{array}{l}\text { Toma } \\
n=35\end{array}$ \\
\hline
\end{tabular}

Valor $p^{*}$

Locus de control

\begin{tabular}{cccccccc} 
Interno & Bajo & 50.3 & 31.6 & 0.006 & 97.7 & 97.1 & 0.570 \\
\hline & Alto & 49.7 & 68.4 & & 2.3 & 2.9 & \\
Externo & Bajo & 44.4 & 40.0 & 0.323 & 54.4 & 62.9 & 0.220 \\
\hline & Alto & 55.6 & 60.0 & & 45.6 & 37.1 &
\end{tabular}

Orientación al rol de género

\begin{tabular}{cccccccc} 
Masculinidad & Baja & 88.3 & 77.2 & 0.023 & 92.8 & 77.1 & 0.004 \\
\hline & Alta & 11.7 & 22.8 & & 7.2 & 22.9 & \\
Feminidad & Baja & 84.5 & 91.2 & 0.126 & 71.9 & 74.3 & 0.467 \\
\hline & Alta & 15.5 & 8.8 & & 28.1 & 25.7 &
\end{tabular}

\begin{tabular}{llllllll} 
Androginia deseable & Baja & 70.2 & 64.9 & 0.257 & 75.9 & 60.0 & 0.031 \\
\hline & Alta & 29.8 & 35.1 & & 24.1 & 40.0 &
\end{tabular}

\begin{tabular}{lllllllll} 
Indiferenciado deseable & Baja & 63.2 & 73.7 & 0.081 & 65.0 & 88.6 & 0.002 \\
\hline Alta & 36.8 & 26.3 & & 35.0 & 11.4 &
\end{tabular}

\begin{tabular}{lllllllll} 
Machismo & Baja & 73.7 & 52.6 & 0.001 & 81.2 & 57.1 & 0.001 \\
\hline Alta & 26.3 & 47.4 & & 18.8 & 42.9 &
\end{tabular}

\begin{tabular}{llllllll} 
Sumisión & Baja & 86.3 & 89.5 & 0.337 & 83.8 & 91.4 & 0.165 \\
\hline & Alta & 13.7 & 10.5 & & 16.2 & 8.6 &
\end{tabular}

\begin{tabular}{llllllll} 
Androginia no deseable & Baja & 73.1 & 71.9 & 0.484 & 75.9 & 74.3 & 0.480 \\
\hline & Alta & 26.9 & 28.1 & & 24.1 & 25.7 &
\end{tabular}

\begin{tabular}{llllllll} 
Indiferencia no deseable & Baja & 40.1 & 57.9 & 0.009 & 35.0 & 51.4 & 0.038 \\
\hline Alta & 59.9 & 42.1 & & 65.0 & 48.6 &
\end{tabular}

\begin{tabular}{lcccccccc} 
Víctima de intento de abuso sexual & No & 97.1 & 98.2 & 0.518 & 93.5 & 88.6 & 0.204 \\
\hline & Sí & 2.9 & 1.8 & & 6.5 & 11.4 &
\end{tabular}

\begin{tabular}{lccccccc} 
Víctima de abuso sexual consumado & No & 99.1 & 100.0 & 0.629 & 97.9 & 94.3 & 0.185 \\
\hline & Sí & 0.9 & 0.0 & & 2.1 & 5.7 &
\end{tabular}

años probablemente estará fumando varios cigarrillos al día para lograr el mismo efecto.

En cuanto al consumo diario de alcohol, en comparación con los resultados de la ENA 2008 del estado de Morelos, ${ }_{17}^{17}$ en esta muestra se encontraron prevalencias menores tanto para hombres como para mujeres.

Este estudio muestra en esta población mayor consumo de tabaco y alcohol en hombres; sin embargo al analizar por orientación al rol de género, se observa que si bien los hombres presentan mayor consumo, las orientaciones "machista", "androginia no deseable" y "masculina" se asocian en la mujer con el uso de estas sustancias. A su vez, en el hombre se asocia la sumisión que actúa como un inhibidor del consumo de tabaco. Estas asociaciones son similares a las encontradas en otro estudio internacional. ${ }^{44}$ 


\section{Cuadro IV}

Factores ASOCIADOS CON EL USO DIARIO DE TABACO EN ESTUDIANTES DE ESCUELAS PÚBLICAS. Morelos, MÉxico, 2004-2005

$\begin{array}{cccc}\text { Población escolar } & \text { General } & \text { Mujeres } & \text { Hombres } \\ & R M & R M & R M \\ & \text { (IC95\%) } & \text { (IC95\%) } & \text { (IC95\%) }\end{array}$

Sexo

\begin{tabular}{cc} 
Femenino & 1 \\
\hline Masculino & $2.15^{\ddagger}$ \\
& $(1.49-3.10)$
\end{tabular}

Edad

\begin{tabular}{lccc} 
Menos de 20 años & $\mathrm{I}$ & $\mathrm{I}$ & $\mathrm{I}$ \\
\hline Tener 20 años o más & $\mathrm{I} .74^{*}$ & $\mathrm{I} .26$ & $\mathrm{I} .57$ \\
& $(1.08-2.80)$ & $(0.92-1.73)$ & $(0.94-2.62)$
\end{tabular}

Zona de residencia

\begin{tabular}{lccc} 
Rural & $\mathrm{I}$ & $\mathrm{I}$ & $\mathrm{I}$ \\
\hline Semiurbana & $\mathrm{I} .33$ & $\mathrm{I} .40$ & $\mathrm{I} .49$ \\
& $(0.97-1.82)$ & $(0.90-2.18)$ & $(0.96-2.29)$ \\
\hline Urbana & $1.96^{*}$ & $2.22^{*}$ & 1.28 \\
& $(1.08-2.80)$ & $(\mathrm{I} .1 \mathrm{I}-4.44)$ & $(0.7 \mathrm{I}-2.02)$
\end{tabular}

Orientación al rol de género

\begin{tabular}{cccc} 
Machismo & $\begin{array}{c}1.63^{*} \\
(1.02-2.60)\end{array}$ & $\begin{array}{c}4.48^{*} \\
(1.69-1 \mathrm{I} .83)\end{array}$ & $\begin{array}{c}1.09 \\
(0.76-1.56)\end{array}$ \\
\hline \multirow{3}{*}{ Sumisión } & & & \\
& 0.65 & 1.43 & $0.22^{*}$ \\
& $(0.32-1.3 \mathrm{I})$ & $(0.39-5.14)$ & $(0.07-0.63)$ \\
\hline
\end{tabular}

\begin{tabular}{lccc}
$\begin{array}{ccc}\text { Androginia no deseable } \\
4.65^{*}\end{array}$ & $1.69 \ddagger$ & & \\
& 1.09 & & \\
& $(1.05-2.72)$ & $(1.74-12.39)$ & $(0.64-1.85)$ \\
\hline
\end{tabular}

Sintomatología depresiva

\begin{tabular}{cccc} 
Alta & 1.57 & 1.32 & $2.91^{*}$ \\
& $(0.94-2.60)$ & $(0.60-2.89)$ & $(1.29-6.53)$ \\
\hline
\end{tabular}

\begin{tabular}{lccc}
$\begin{array}{l}\text { Locus de control } \\
\text { Externo }\end{array}$ & $1.59 *$ & 1.54 & 1.18 \\
& $(1.10-2.29)$ & $(0.80-2.96)$ & $(0.84-1.65)$ \\
\hline
\end{tabular}

Víctima de intento de abuso sexual

$\begin{array}{llll}\text { Sí } & 1.80 & 4.35 * & 1.60\end{array}$

$(0.83-3.87) \quad(1.54-12.26) \quad(0.73-3.49)$

$* p<0.05$

$\ddagger p<0.001$

\section{Cuadro V}

FActores Asociados CON EL USO REgUlar DE ALCOHOL EN ESTUDIANTES DE ESCUELAS PÚBLICAS. Morelos, MéXıco, 2004-2005

$\begin{array}{cccc}\text { Población escolar } & \text { General } & \text { Mujeres } & \text { Hombres } \\ & R M & R M & R M \\ & \text { (IC95\%) } & \text { (IC95\%) } & \text { (IC95\%) }\end{array}$

Sexo

$\begin{array}{cc}\text { Femenino } & 1 \\ \text { Masculino } & 3.22^{*} \\ & (2.07-5.00)\end{array}$

Edad

\begin{tabular}{lccc} 
Menos de 20 años & $\mid$ & $\mid$ & $\mid$ \\
Tener 20 años o más & $2.47^{*}$ & 1.97 & $3.34^{*}$ \\
& $(I .4 I-4.3 \mathrm{I})$ & $(0.98-3.95)$ & $(I .80-6.29)$ \\
\hline
\end{tabular}

Zona de residencia

Rural I I ।

$\begin{array}{llll}\text { Semiurbana } & 1.23 & 1.05 & 2.11^{*}\end{array}$

\begin{tabular}{cccc} 
& $(0.92-1.65)$ & $(0.37-2.96)$ & $(\mathrm{I} .30-3.4 \mathrm{I})$ \\
\hline Urbana & $2.33^{*}$ & $\mathrm{I} .76$ & $\mathrm{I} .55^{*}$ \\
& $(\mathrm{I} .27-4.25)$ & $(0.72-4.27)$ & $(\mathrm{I} .05-2.29)$ \\
\hline
\end{tabular}

Orientación al rol de género

$\begin{array}{llll}\text { Masculinidad } & 2.72^{*} & 3.90^{*} & 1.52\end{array}$

$(1.17-6.36) \quad(1.18-12.8 I) \quad(0.70-3.29)$

Locus de control

\begin{tabular}{lccc} 
Interno & $\begin{array}{c}1.85^{*} \\
(1.04-3.30)\end{array}$ & $\begin{array}{c}1.50 \\
(0.60-3.73)\end{array}$ & $\begin{array}{c}1.92^{*} \\
(1.03-3.58)\end{array}$ \\
\hline \multirow{3}{*}{ Externo } & & & \\
& $2.05^{*}$ & 1.96 & 1.34 \\
& $(1.19-3.53)$ & $(0.96-3.91)$ & $(0.92-1.95)$ \\
\hline
\end{tabular}

En la escala IMAFE ${ }^{51}$ los reactivos de "machismo" y "androginia no deseable" comprenden comportamientos rebeldes y antisociales que aparecen señalados en estudios en población adolescente sobre las características percibidas de las personas fumadoras. ${ }^{59}$ Esta asociación entre tabaquismo y rebeldía en mujeres jóvenes se había reportado desde los años ochenta y noventa en estudios poblacionales: ${ }^{60}$ rebeldía; ${ }^{61,62}$ rebeldía y rechazo a la autoridad de los adultos; ${ }^{63}$ menor obligación con normas paternas; ${ }^{64}$ tolerancia a la desviación ${ }^{65}$ y delincuencia. ${ }^{66}$ Se ha discutido que estos comportamientos machistas o rebeldes asociados con el tabaco pudieran expresar una forma de empoderamiento de las jóvenes para obtener independencia y poder, demostrar competencias socia- 
les y culturales sobre todo en zonas urbanas con mayor influencia de los medios de comunicación y nuevos estilos de vida. ${ }^{67}$ Algunos teóricos describen al "machismo" como un manejo de apariencias. ${ }^{68}$ Este puede ser reforzado por la mercadotecnia de las tabacaleras que promueven comportamientos rebeldes asociados con el hábito de fumar ${ }^{69,70}$ y no solamente la obtención de glamour en mensajes dirigidos hacia la población femenina joven. ${ }^{71}$ Un factor señalado es el uso de imágenes audaces con el propósito de que las jóvenes identifiquen la propia identidad personal con la "personalidad audaz" y "rebelde" de la marca de cigarrillos. ${ }^{72}$ En cuanto a la "androginia no deseable", ésta se asocia también con el consumo de tabaco porque la persona tiene una menor capacidad de desenvolvimiento social, menor habilidad para expresar emociones y empatía con las emociones de los otros, más estrés y neurosis, mayor victimización y perpetración de violencia. ${ }^{73}$ La sumisión en el hombre es inhibitoria por la pasividad del rol, la dependencia y aprobación social.

En cuanto al manejo emocional con tabaquismo, se ha señalado que las mujeres fuman para enfrentar experiencias negativas, ${ }^{74}$ como el intento de abuso sexual señalado en otros estudios. ${ }^{75-77}$ En hombres, el enfrentar la depresión fumando ha sido señalado previamente, ${ }^{78-81}$ explicándose que en aras de hacer del hombre el principal proveedor económico del hogar, en la división sexual del trabajo se ha reprimido culturalmente la expresión tanto de emociones como de depresión, lo que lo lleva a liberarse emocionalmente mediante las adicciones. ${ }^{82}$

Por otra parte, con la asociación entre alcohol y orientación a la masculinidad en la mujer, ésta se orienta a un mayor logro de metas profesionales, evitando intimidad y expresividad emocional, lo que puede motivarla a recurrir al alcohol, al igual que el hombre con el tabaco, para el manejo emocional. Además, en el ambiente social de las jóvenes prevalece una mezcla de actitudes y creencias de género tradicionales e igualitarias que desestabilizan el proyecto de vida independiente al confrontar el ejercicio profesional con la formación de una familia. ${ }^{83}$

En los hombres la no asociación entre alcohol y machismo pudiera explicarse por la diversidad en sus orientaciones al rol de género: más altos en "androginia positiva", "androginia negativa", "machismo" y "masculinidad". Esto puede representar un cambio cultural y social en los roles de género en el hombre joven de Morelos, con respecto a comunidades más tradicionales, ${ }^{84}$ y pudiera ser que el alcoholismo no sea la expresión del machismo en los nuevos jóvenes urbanos.

En general se observa que tanto para mujeres como para hombres, el locus de control externo se asocia con el consumo regular de alcohol por la dependencia social de aprobación, ${ }^{85}$ sin embargo, en hombres el locus interno incrementa la probabilidad de uso de alcohol a pesar de que se ha señalado como un factor protector que se incrementa con la recuperación del alcoholismo. ${ }^{86} \mathrm{~A}$ pesar de ello, recientemente las compañías vinícolas han elaborado publicidad que promueve el consumo responsable de alcohol y la idea de que el bebedor "tiene el control" y "decide cuánto beber". Estos mensajes asocian locus de control interno con consumo y pretenden acercar la imagen social del bebedor a la de un "consumidor responsable" alejándola de la imagen del "enfermo alcohólico". Estos mensajes pudieran reforzar la falsa creencia de tener control personal sobre el propio alcoholismo y de poder dejar de beber cuando la persona lo desee.

\section{Conclusiones}

Los resultados de este estudio sugieren que si bien el hombre es el principal consumidor de alcohol y tabaco, las orientaciones de género aumentan o disminuyen diferencialmente la probabilidad de consumo. Comportamientos machistas y de masculinidad con fuerte arraigo cultural en México son adoptados también por las jóvenes como expresión de rebeldía o logro en asociación con el consumo de alcohol y tabaco, respectivamente. En los hombres de esta muestra no se encontró asociación entre "machismo" y alcoholismo y sí asociación entre "sumisión" y depresión con tabaquismo. Los roles "andrógino positivo" y "feminidad" no se asociaron con las adicciones. En general el locus de control externo incrementa el consumo de alcohol y tabaco, y el interno el alcoholismo en el hombre. En zonas urbanas se incrementa el consumo de tabaco en la mujer y el de alcohol en el hombre.

Es importante intervenir contra las adicciones mediante programas, cuidando en no usar estereotipos de género para el diseño de los mismos, como suponer que la mujer no pueda asumir comportamientos adictivos, machistas o de masculinidad o sumisión en el hombre. Incorporar en los mensajes que las adicciones y el machismo no son un medio para el empoderamiento, madurez e independencia, y fomentar en talleres el desarrollo de habilidades de expresividad emocional y de realización de metas. Es decir, el desarrollo de la "androginia positiva" sin distinción de género para prevenir las adicciones.

Finalmente, se debe incorporar el estudio de los roles de género en el estudio de las adicciones y la perspectiva de género debe reconocer los problemas emergentes de las nuevas mujeres y también la problemática de los hombres.

Declaración de conflicto de intereses. Los autores declararon no tener conflicto de intereses. 


\section{Referencias}

I. de Vries H, Engels RCME, Kremers S, Wetzels J, Mudde A. Parents and friends smoking status as predictors of smoking onset: Findings from six European Countries. Health Educ Res 2003;18(5):627-636.

2. Abreu AM, de Lima JM, Matos LN, Pillon SC. Alcohol use and traffic accidents: a study of alcohol levels. Rev Lat Am Enfermagem 2010;18 Spec No:5 I3-520.

3. Secretaría de Educación Pública, Instituto Mexicano de Psiquiatría. Encuesta Nacional sobre el Uso de Drogas entre la Comunidad Escolar Nacional. México, DF: SEP, IMP, 1993.

4. Villatoro J, Medina-Mora ME, Hernández M, Fleiz C, Amador N, Bermúdez P. La encuesta de estudiantes de nivel medio y medio superior de la Ciudad de México: Noviembre 2003. Prevalencias y evolución del consumo de drogas. Salud Mental 2005; 28(I):38-5I.

5. Alati R, Kinner S, Najman JM, Fowler G, Watt K, Green D. Gender Differences in the Relationships Between Alcohol Tobacco and Mental Health in Patients Attending an Emergency Department. Alcohol Alcohol 2004;39(5):463-469.

6. Inegi, SSA, Conadic, IMP, DGE. Encuesta Nacional de Adicciones 2002, INEGI 2004. [consultado agosto 2010] Disponible en: http://200.23.8.5/ prod_serv/contenidos/espanol/bvinegi/productos/continuas/sociales/ salud/2004/Ena02.pdf

7. SEP-IMP. Encuesta Nacional sobre uso de Drogas entre la Comunidad Escolar. México: SEP-IMP 1976.

8. SEP-IMP. Encuesta Nacional sobre Uso de Drogas entre la Comunidad Escolar. México: SEP-IHP 199I.

9. Fleiz C, Borges G, Rojas E, Benjet C, Medina-Mora ME. Uso de Alcohol, Tabaco y Drogas en Población Mexicana, un estudio de cohortes. Salud Mental 2007; 30(5):63-73.

10. Valdés-Salgado R, Reynales-Shigematsu LM, Lazcano-Ponce E, Hernández-Avila M. Antes y después del Convenio Marco en México: una comparación desde la Encuesta sobre Tabaquismo en Jóvenes 2003 y 2006. Salud Publica Mex 2007; 49(suppl.2):sI55-s169.

II. SSa. Dirección General de Epidemiología: Encuesta Nacional de Adicciones. México DF: SSa. 1993.

12. Brzakowski M, Lourdel E, Cabry R, Oliéric MF, Claeys C, Devaux A, et al. Épidémiologie du couple infertile. Journal de Gynécologie Obstétrique et Biologie de la Reproduction 2009 38(SI-2):F3-7.

13. Salamó Avellaneda A, Gras Pérez ME, Font-Mayolas S. Alcohol consumption patterns in adolescence. Psicothema 2010;22(2):189-195. 14. Delegación del Gobierno para el Plan Nacional sobre Drogas. Informe de la Encuesta Estatal sobre Uso de Drogas en Estudiantes de Enseñanzas Secundarias (ESTUDES) 2008. Madrid, España: Ministerio de Sanidad y Política social, 2009.

15. WHO. Health and Health Behaviour among Young People. Copenhagen: WHO, 2000.

16. Avellaneda AS, Gras PE, Font-Mayolas S. Patrones de consumo de alcohol en la adolescencia. Psicothema 2010 22(2): 189-195.

17. Instituto Nacional de Salud Pública. Encuesta Nacional de Adicciones 2008. Cuernavaca, Morelos, México: Instituto Nacional de Salud Pública, 2009.

18. Rasch V. Cigarette, alcohol, and caffeine consumption: risk factors for spontaneous abortion. Acta Obstet Gynecol Scand 2003;82(2):182-188.

19. Keegan J, Parva M, Finnegan M, Gerson A, Belden M. Addiction in pregnancy. J Addict Dis 2010;29(2):175-191.

20. Ripabelli G, Cimmino L, Grasso GM. Alcohol consumption, pregnancy and fetal alcohol syndrome: implications in public health and preventive strategies. Ann Ig 2006; 18(5):39|-406.

21. Salihu HM, Kornosky JL, Lynch O, Alio AP, August EM, Marty PJ. Impact of prenatal alcohol consumption on placenta associated syndromes. Alcohol 2010 Jun 30 [Epub ahead of print].
22. Li Cl, Chlebowski RT, Freiberg M, Johnson KC, Kuller L, Lane $\mathrm{D}$, Prentice R, et al. Alcohol Consumption and Risk of Postmenopausal Breast Cancer by Subtype: the Women's Health Initiative Observational Study. J Natl Cancer Inst 2010; 23.

23. Allen NE, Beral V, Casabonne D, Kan SW, Reeves GK, Brown A, et al. Million Women Study Collaborators. Moderate alcohol intake and cancer incidence in women. J Natl Cancer Inst 2009; 10I(5):296-305. 24. Department of Health and Human Services. Women and smoking. A report of the Surgeon General. Washingtomn DC: U.S. Dept. of Health and Human Services, Public Health Service, 200I.

25. Windham GC, Mitchell P, Anderson M, Lasley BL. Cigarette smoking and effects on hormone function in premenopausal women. Environ Health Perspect 2005; I I3(I0): 1285-1290.

26. Cooper AR, Moley KH. Maternal tobacco use and its preimplantation effects on fertility: more reasons to stop smoking. Semin Reprod Med 2008;26(2):204-2/2.

27. Kinney A, Kline J, Levin B. Alcohol, caffeine and smoking in relation to age at menopause. Maturitas 2006;54(I):27-38.

28. Roelands J, Jamison MG, Lyerly AD, James AH. Consequences of smoking during pregnancy on maternal health. J Womens Health (Larchmt) 2009; I8(6):867-872.

29. Bruchova H, Vasikova A, Merkerova M, Milcova A, Topinka J, Balascak I, et al. Effect of maternal tobacco smoke exposure on the placental transcriptome. Placenta 2010;3I(3):186-191.

30. Aliyu MH, Lynch O, Wilson RE, Alio AP, Kristensen S, Marty $\mathrm{PJ}$, et al. Association between tobacco use in pregnancy and placentaassociated syndromes: a population-based study. Arch Gynecol Obstet 2010; 31.

31. Foidart JM, Seak-San S, Emonts P, Schaaps JP. Vascular placental pathology in high-risk groups: definition and synopsis. Ann Med Interne (Paris) 2003;154(5-6):332-339.

32. Coutinho PR, Cecatti JG, Surita FG, de Souza JP, de Morais SS. Factors associated with low birth weight in a historical series of deliveries in Campinas, Brazil. Rev Assoc Med Bras 2009;55(6):692-699.

33. Lavezzi AM, Corna MF, Matturri L. Ependymal alterations in sudden intrauterine unexplained death and sudden infant death syndrome: possible primary consequence of prenatal exposure to cigarette smoking. Neural Dev 2010 19;5:17.

34. Prescott E, Osler M, Andersen PK, Hein HO, Borch-Johnsen K, Lange $P$, et al. Mortality in women and men in relation to smoking. Int J Epidemiol 1998;27(I):27-32.

35. Gaur DS, Taleker MS, Pathak VP. Alcohol intake and cigarette smoking: impact of two major lifestyle factors on male fertility. Indian J Pathol Microbiol 2010;53(I):35-40.

36. Li Y, Lin H, Li Y, Cao J. Association between socio-psycho-behavioral factors and male semen quality: systematic review and meta-analyses. Fertil Steril 2010 Jul 30 \{Epub ahead of print)

37. Kupelian V, Link CL, McKinlay JB. Association between smoking, passive smoking, and erectile dysfunction: results from the Boston Area Community Health (BACH) Survey. Eur Urol 2007;52(2):416-422. 38. Millett C, Wen LM, Rissel C, Smith A, Richters J, Grulich A, et al. Smoking and erectile dysfunction: findings from a representative sample of Australian men. Tob Control 2006;15(2):136-139.

39. de Vries H, Mudde A, Leijs I, Charlton A, Vartiainen E, Buijs G, et al. The European Smoking prevention framework approach (EFSA): An example of integral prevention. Health Edu Res 2003;18(5):6I I-626. 40. Caballero R, Madrigal DME, Hidalgo SMA, Villaseñor A. El Consumo de tabaco, alcohol y drogas ilegales en los jóvenes de diferentes estratos socioeconómicos en Guadalajara. Salud Mental 1999;(22)4: I-8.

4I. Poulin C, Hand D, Boudreau B, Santor D. Gender differences in the association between substance use and elevated depressive symptoms in a general adolescent population. Addiction 2005;(1000):525-535. 
42. Shadel WG, Niaura R, Abrams DB. Adolescent's response to the gender valence of cigarette advertising imagery: The role of affect and the self-concept. Addict Behav 2004; (29): 1735-1744.

43. Huselid FR, Cooper, LM. Gender Roles as mediators of sex differences in adolescent alcohol use and abuse. J Health Soc Behav 1992;(33):348-362.

44. Emselie $\mathrm{C}$, Hunt $\mathrm{K}$, Macintyre $\mathrm{S}$. How similar are the smoking and drinking habits of men and women in non-manual jobs? Eur J Public Health 2002; I2(I):22-28.

45. Bem SL. Gender Schema Theory: A cognitive account of sex typing. Psychological Review 1981;88:354-364.

46. Arillo-Santillan E, Lazcano-Ponce E, Hernandez-Avila M, Fernandez $\mathrm{E}$, Allen $\mathrm{B}, \mathrm{Valdes} \mathrm{R}$, et al. Associations between individual and contextual factors and smoking in 13293 mexican students. Am J Prev Med 2005;28(I):4I-5I.

47. Bronfman M, Guiscafré H, Castro V, Castro R, Gutiérrez G. II La medición de la desigualdad: una estrategia metodológica, análisis de las características socioeconómicas de la muestra. Arch Invest Med Mex 1988; 19:35I-360.

48. INEGI. Dirección General de Geografía. Comité de Cartografía Censal. Tomo I. Aguascalientes, México: INEGI, 1987.

49. OPS, INSP. Encuesta Global de Tabaquismo en Adultos. México 2009. Cuernavaca (México): Instituto Nacional de Salud Pública, 20I0/Organización Panamericana de la Salud, 2010.

50. Bastos Fl, Bertoni N, Hacker MA. Consumo de alcohol e drogas: principais achados de pesquisa de âmbito nacional, Brasil 2005. Rev Saùde Pùblica 2008; 42(SI):109-117.

5I. Lara-Cantú MA. Inventario de Masculinidad y Feminidad (IMAFE). México: El Manual Moderno, 1993.

52. Jiménez-Guzmán ML. Algunas ideas acerca de la construcción social de las masculinidades y las feminidades, el mundo público y el mundo privado. En: Jiménez-Guzmán ML, Tena Guerrero $O$. Reflexiones sobre masculinidades y empleo. Cuernavaca: UNAM-CRIM 2007.

53. Verduzco MA, Lara-Cantú MA, Acevedo M, Cortés J. Validación del Inventario de Autoestima de Coopersmith para niños mexicanos. Revista Intercontinental de Psicología y Educación 1994;7(2):55-64.

54. Zung WWK. A self-rating depression scale. Arch Gen Psychiatry 1965;12:63-70.

55. La Rosa. Locus de contrôle: Uma escala de avaliacao. Psicología: Teoría e Pesquisa 1991;7(3) :327-344.

56. Russell DE. The incidence and prevalence of intrafamilial and extrafamilial sexual abuse of female children. Child Abuse Negl I983;7(2):I33-I46. 57. Wyatt GE. The sexual abuse of Afro-American and white-American women in childhood. Child Abuse Negl 1985;9(4):507-519.

58. Hosmer DW, Lemenshow S. Applied logistic regression. Nueva York: John Wiley \& Sons, 1989.

59. Luke D, Allen P, Arian G, Crawford M, Headen S, Spigner AC, et al. Teen's images of smoking and smokers. Public Health Rep 200 I; II6 Suppl I:194-202.

60. Women and Smoking: A Report of the Surgeon General. Office on Smoking and Health (US). Atlanta (GA): Centers for Disease Control and Prevention (US), 200I.

6I. Pierce JP, Farkas A, Evans N, Berry C, Choi W, Rosbrook B, et al. Tobacco Use in California 1992: A Focus on Preventing Uptake in Adolescents. Sacramento (CA): California Department of Health Services, 1993. 62. Sussman S, Dent CW, Flay BR, Hansen WB, Johnson CA. Psychosocial predictors of cigarette smoking onset by white, black, Hispanic, and Asian adolescents in Southern California. Morb Mort Week Rep 1987; 36(Suppl 4):IIS-16S

63. Best JA, Brown KS, Cameron R, Manske SM, Santi S. Gender and predisposing attributes as predictors of smoking onset: implications for theory and practice. J Health Educ 1995;26(Suppl 2):S52-S60.
64. Skinner WF, Massey JL, Krohn MD, Lauer RM. Social influences and constraints on the initiation and cessation of adolescent tobacco use. J Behav Med 1985; 8(4): 353-376.

65. Chassin L, Presson CC, Sherman SJ, Corty E, Olshavsky RW. Predicting the onset of cigarette smoking in adolescents: a longitudinal study. J App Soc Psych 1984; 14(3):224-243.

66. Stanton WR, Lowe JB, Silva PA. Antecedents of vulnerability and resilience to smoking among adolescents. J Adoles Health 1995; 16(I):71-77. 67. Haines RJ, Poland BD, Johnson JL. Becoming a 'real' smoker: cultural capital in young women's accounts of smoking and other substance use. Sociol Health IIIn 2009;3I(I):66-80.

68. Ramírez-Hernández FA. Violencia masculina en el hogar. México: Pax, 2002.

69. Hendlin Y, Anderson SJ, Glantz SA. 'Acceptable rebellion': marketing hipster aesthetics to sell Camel cigarettes in the US. Tob Control 2010;19(3):213-222.

70. Kaufman NJ, NichterM. The marketing of tobacco to women: global perspectives. En: Samet JM, Yoon S-Y, eds. Women and the tobacco epidemic. Challenges for the 21 st century. Ginebra: World Heath Organization, 2001;69-98.

7I. Knight J, Chapman S. "Asian yuppies...are always looking for something new and different": creating a tobacco culture among young Asians. Tob Control 2004; 13 Suppl 2:ii22-29.

72. Cortese DK, Lewis MJ, Ling PM. Tobacco Industry Lifestyle Magazines Targeted to Young Adults. J Adolesc Health 2009;45(3):268-280.

73. Bem SL. The measurment of psychological androgyny. J Consult Clin Psychol 1974;(42)2: I55-162.

74. Fernández E, Schiaffino A, Peris M. Tabaquismo en mujeres:un problema de salud emergente. Enf Emerg 200I;3(4):184-190.

75. Clark DB, Thatcher DL, Martin CS. Child abuse and other traumatic experiences, alcohol use disorders, and health problems in adolescence and young adulthood. J Pediatr Psychol 2010;35(5):499-5I0.

76. Sousa S, Correia T, Ramos E, Fraga S, Barros H. Violence in adolescents: social and behavioural factors. Gac Sanit 2010;24(I):47-52.

77. Choudhary E, Coben JH, Bossarte RM. Gender and time differences in the associations between sexual violence victimization, health outcomes, and risk behaviors. Am J Mens Health 2008;2(3):254-259.

78. Luk JW, Tsoh JY. Moderation of gender on smoking and depression in Chinese Americans. Addict Behav 2010;35(II):1040-1043.

79. Brown C, Madden PA, Palenchar DR, Cooper-Patrick L. The association between depressive symptoms and cigarette smoking in an urban primary care sample. Int J Psychiatry Med 2000;30(I):15-26.

80. John U, Meyer C, Rumpf HJ, Hapke U. Smoking, nicotine dependence and psychiatric comorbidity--a population-based study including smoking cessation after three years. Drug Alcohol Depend 2004;76(3):287-295.

8I. John U, Meyer C, Rumpf HJ, Hapke U. Depressive disorders are related to nicotine dependence in the population but do not necessarily hamper smoking cessation. J Clin Psychiatry 2004;65(2):169-176.

82. Farell W. The Myth of Male Power. Berkley: New York, 1994.

83. Petit VM, Prat TM. Prevención de la violencia de género en la adolescencia. Barcelona: Icaria, 201I.

84. Contreras-Urbina JM. La legitimidad social de la violencia contra las mujeres en la pareja. Un estudio cualitativo con varones en la ciudad de México. En: Castro R, Cacique I, Estudios sobre cultura, género y violencia contra las mujeres. Cuernavaca: CRIM-UNAM, 2008.

85. Yeh MY. Measuring readiness to change and locus of control belief among male alcohol dependent patients in Taiwan: comparision of the different degrees of alcohol dependence. Psychiatry Clin Neurosci 2008; 62(5):533-539.

86. Suresh Kumar PN, Thomas B. Family intervention therapy in alcohol dependence syndrome: One year follow up study. Indian J Psychiatry 2007;49:200-204. 